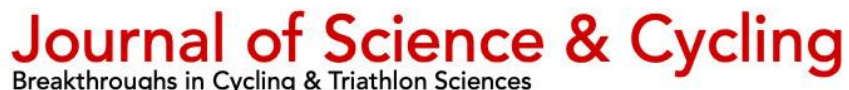

Article

\section{Effects of ergonomic clip-on handles on upper-body vibration transmissibility and muscular activity during pedalling with vibrations}

\author{
Sébastien Duc ${ }^{1}$, Victor Scholler ${ }^{1}$, Frédéric Puel ${ }^{1}$, Geoffrey Millour ${ }^{1}$, William Bertucci ${ }^{1}$ \\ 1. Laboratory of Performance, Health, Metrology and Society (EA7507), University of Reims Champagne- \\ Ardenne, Reims, France
}

* Correspondence: (SD) sebastien.duc@univ-reims.fr

Received: 12 June 2020; Accepted: 30 June 2021; Published: 30 June 2021

\begin{abstract}
Mountain bikers often report impaired finger sensitivity caused by mechanical vibrations and misalignment between the wrist and the forearm when using traditional (cylindrical) handles. The aim of this study was to evaluate the acute effects of ergonomic clip-on handles that allowed the hand to rest on the medial carpal bone, on muscular activity, vibration transmissibility between the cycle ergometer and body segments, and handgrip strength. Sixteen cyclists performed two pedalling exercises at $\sim 200 \mathrm{~W}$ lasting 20 minutes on a cycle ergometer that delivered vibrations under the fork (vertical amplitude: 4-25 mm; frequency: $417 \mathrm{~Hz}$ ) whilst using cylindrical handles and ergonomic clip-on handles with a randomized order. Compared to cylindrical handles, ergonomic clip-on handles decreased significantly vibration transmissibility to the extensor digitorum, triceps brachii and flexor carpi radialis muscles by 10, 10 and $7 \%$, respectively. The surface electromyography activity of the flexor carpi radialis decreased by $45 \%$, while that of the triceps brachii increased by $12 \%$ (both significantly). Unlike the cylindrical handles, the ergonomic clip-on handles did not involve a significant decrease in the maximal handgrip force after the pedalling exercise. The ergonomic clip-o handles may prevent symptoms of hand-arm vibration syndrome in mountain bikers and could preserve their ability to effectively maneuver and brake the bike.
\end{abstract}

Keywords: mountain biking, ulnar nerve syndrome, ergonomic handle, vibrations, electromyography, handgrip force

\section{Introduction}

Mountain biking (MTB) is both a recreational activity and a competitive sport composed of sub-disciplines, including notably cross-country (XC) and downhill biking. XC biking involves riding on varied terrain, including rocky and dirt paths, technical single tracks, and open forest roads (Lee et al. 2002). Because of the irregularities of the ground, mountain bikers are often subjected to repetitive and continuous mechanical vibrations. These vibrations are mainly directed to the vertical axis, are characterised by low frequencies (i.e., $<50 \mathrm{~Hz}$ ) (De Lorenzo and Hull 1999; Levy and Smith 2005), and are transmitted to the body from the pedals, saddle and handlebar. Vibrations can be partly reduced by the use of suspension (Faiss et al. 2007; Levy and Smith 2005), low tyre pressure (Tarabini et al. 2015), large tyres (Macdermind et al. 2015), padded saddles, silicone grips, and bike gloves with foam or gel elements (Bertucci and Chiementin 2013). Certain body parts, particularly the arms and legs (Wang et Hull 1997), and soft tissues at the interface between the bike and the body, also play a role in the dampening mechanism

(c) 2021 fist author, licensee JSC. This is an Open Access article distributed under the terms of the Creative Commons Attribution License ((http://creativecommons.org/licenses/by/4.0/) which permits unrestricted use, distribution, and reproduction in any medium, provided the original work is properly cited. 
(Issurin 2005; Mester et al. 1999). Soft tissues vibrate in direct response to mechanical excitation and thus reduce the vibrational energy to a certain extent (Wakeling et al. 2002). Several studies (Abercromby et al. 2007; Hazell et al. 2007; Issurin 2005; Mester et al. 1999) have reported an increase in muscle activity during vibration exercise as a result of dampening, which possibly reflects an increase in motor unit recruitment for a given force produced (Mester et al. 1999).

Mountain bikers often complain of tingling, numbness, impaired sensitivity and/or limited dexterity in the fingers and hands during and sometimes after riding their bicycle (Akuthota et al. 2005; Capitani and Beers 2002). They are commonly referred to the ulnar tunnel syndrome, which is caused by a chronic pressure applied to the area of the hand on the handlebar (Steward 1993). This phenomenon is more exacerbated in mountain bikers than in road cyclists because their forearms are more pronated when they grip cylindrical handles. Moreover, compared to road bikes, mountain bikes are always equipped with wider handlebars to improve handling, which can induce misalignment of the wrists with the forearms. Consequently, ulnar nerve medial compression in Guyon's canal could increase, notably during long distance MTB rides (Capitani and Beer, 2002). In addition, as the distance between the two handles exceed shoulder width, the lateral muscles of both the hands and forearms can be more stretched and activated, which can enhance muscular fatigue and mechanical strains to tendons and ligaments of the wrist. Furthermore, the common use of a standing position during MTB downhill sections leads to high pressure to the hands because much of the body weight is supported by the upper limbs (Capitani and Beer, 2002).

Change in the sensation in the fingers are also a characteristics of hand-arm vibration syndrome, which occurs in individual who suffer constant or repeated shocks to the base of the palm, such as workers who use vibrating tools (Radwin et al. 1987). Chronic vibration exposure combined with the occurrence of ulnar tunnel syndrome while mountain biking can result in reduced fingers mobility, pain in the hands and arms, and impaired grip force (Radwin et al. 1987). Hand grip force ability is particularly important to the context of MTB for braking and maneuvring the bike during steep downhills and technical sections of a track.

Specific handlebars and handles for preventing symptoms of ulnar tunnel syndrome have been recently developped. For example, MTB companies have designed handlebars with upsweep and backsweep angles to raise the hands and bring them closer to the trunk to prevent misalignment of the wrist, thus limiting both ulnar nerve compression and medial adduction of the wrists. Other companies have proposed ergonomic handles with a larger support areas at the lateral side of the cylindrical handles to relieve hand pressure and provide maximum control (e.g.., Contour XC grips, Specialized; GP MTB Comfort Series, Ergon Bike Ergonomics; Grips Ergonomic GR-S22, Xcellent Bike Components). Ergonomic clip-on handles (e.g. SPIRGRIPS ${ }^{\circledR}$, Geneva, Switzerland) have been developed to both modify the position of the hands and forearms and to reduce the distance between the hands and the shoulders. They offer a second support area on the handlebar in addition to the cylindrical handles (Figure 1). These ergonomic clip-on handles have been authorised by the Union Cycliste Internationale to be used during MTB competitions. According to the manufacturer, these clip-on handles were designed to correct the position of the hands, naturally aligning the joints of the forearms by slightly externally rotating the wrist. Consequently, the hands rest on the medial carpal bone and the mechanical constraints between the scaphoid and the radius are decreased (Figure 2). 


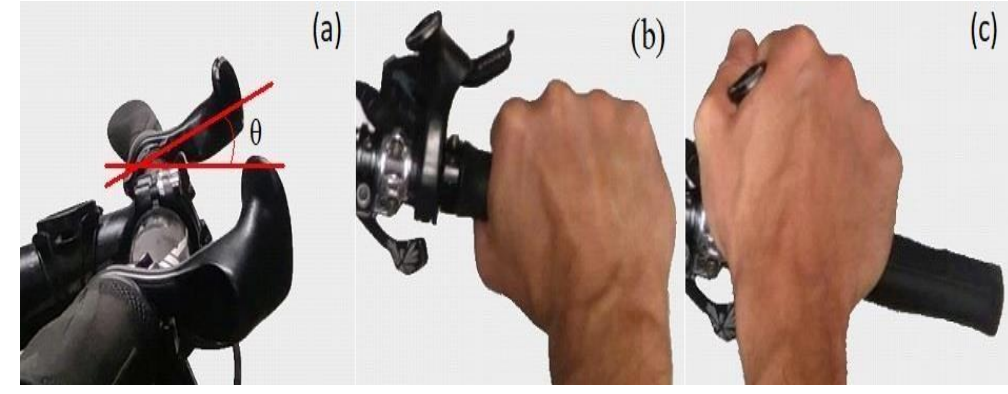

Figure 1: Illustration of the inclination of the ergonomic handle with respect to the horizontal line (a), the hand position on the cylindrical handle (b), and the hand position on the ergonomic handle (c)

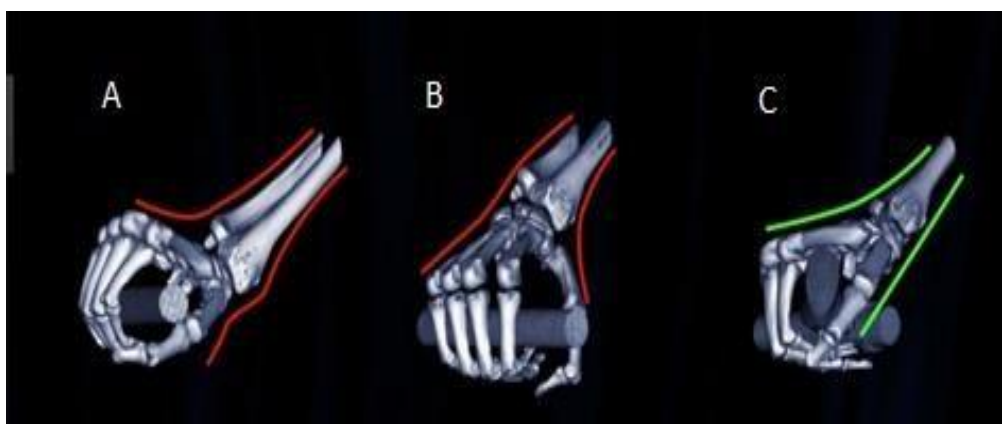

Figure 2: X-ray representation of the alignment between the bone forearm and the hand with the use of a cylindrical handle (A), an ergonomic handle with an external support area $(B)$ and an ergonomic clip-on handle $(C)$. This figure was provided by the SPI

Considering the changes in the forearmhand configuration and support described above, the ergonomic clip-on handles may modify the muscular activity of the arms and the vibration transmissibility to the upper body. Some studies (De Ponte et al. 2015; Di Domizio and Keir 2010) have indeed shown that forearm extensor muscle activity is higher when the forearm is pronated than when it is in a neutral or supinated posture during grip tasks. In addition, other studies (Aldien et al. 2005; Xu et al. 2017) have shown that handle size, hand-arm posture and hand forces affect the magnitude of vibrational energy absorbed by the hand-arm system.

The aim of this study was to evaluate the effects of the SPIRGRIPS ${ }^{\circledR}$ handles on vibration transmissibility and muscular activity during pedalling with vibrations. We hypothesized that these ergonomic clip-on handles induce lower vibration transmissibility and muscle activity in both the forearm and arm than do traditional (cylindrical) handles due to differences in the hand and forearm configuration. We also supposed that the handgrip force decreases c) less after a prolonged pedalling exercise when SPIRGRIPS ${ }^{\circledR}$ handles are used compared to when traditional handles are used since they theoretically prevent ulnar nerve medial compression.

\section{Materials and Methods}

\section{Participants}

Sixteen trained cyclists, including fourteen men (age: $26.8 \pm$ 9.9 years old; height: $1.79 \pm 0.05 \mathrm{~m}$; mass: $70.0 \pm 5.9 \mathrm{~kg}$ ) and two women (age: $27.5 \pm 7.5$ years old; height: $1.69 \pm 0.02 \mathrm{~m}$; mass: $63.5 \pm 6.5 \mathrm{~kg}$ ), volunteered to participate in the study. All participants had at least three years of cycling experience, and they regularly performed MTB training and engaged in MTB competitions. None of the participants had prior experience with ergonomic clip-on handles, nor had they suffered from hand or finger palsy or ulnar neuropathy. Before the data collection began, they provided written informed consent after having been informed of the potential risks and benefits of the study. The study was approved by the University of Reims Champagne-Ardenne's ethics committee of biomedical research and was conducted in accordance with the Declaration of Helsinki with ethical guidelines (Harris and Atkinson 2011).

\section{Protocol}

Each cyclist performed a one-day laboratory test that included two pedalling exercises lasting 20 minutes each with (1) traditional handles (TH) and (2) ergonomic clip-on handles (EH). According to the manufacturer guidelines, all EH were positioned between the brake levers and the gear triggers (Figure 1). The participants selfselected the inclination of the EH in the sagittal plane on the basis of their comfort. The inclination angles of the right and left $\mathrm{EH}$, which were measured with respect to the horizontal plane with an inclinometer (Plurimeter, Rippstein, Switzerland), were 
always positive and between $15^{\circ}$ and $35^{\circ}$ (Figure 1). The support part of the EH used in the current study was not covered with a soft material (i.e., silicone or foam). The $\mathrm{TH}$ were cylindrical in shape (outer diameter $=30$ $\mathrm{mm}$ ) and covered by gum rubber (Ergon Technical GE1 EVO grip, Ergon Bike Ergonomics, Kolenz, Germany). The two experimental conditions were performed in a random order.

All the cyclists used the same aluminum hardtail mountain bike (Big. Seven 40 White model, 2015, Merida, Taiwan) equipped with aluminum stem and handlebars. The rear wheel was fixed to an electromagnetically braked ergometer (Super Crono, Elite, Fontaniva, Italy) with a quick-release skewer (Figure 3). The pressure of the rear tyre was set to 2 bars and was checked before each test. In addition, the pressure between the ergometer roller and the rear tyre was standardized by turning the screw by 540 degrees when the ergometer roller was in contact with the tyre. The aluminum suspension fork (RockShox XC $30,100 \mathrm{~mm}$ of vertical displacement, Sram LLC, Chicago, USA) was attached to a mechanical pivot driven by an electrical motor (UNEL MEC, MCR components, Milano, Italy) coupled to a frequency converter (Rofre 897, Helmut Rossmanith $\mathrm{GmbH}$, Uhingen, Germany) (Figure 3). This bespoke cycle ergometer allowed mechanical vibrations to be generated from the pivot and transmitted to the front fork during pedalling. As we did not use a rigid fork, we decided to inflate the suspension fork to 2 bars in order to minimize vibration damping between the mechanical pivot and the handlebar, and to block the suspension by closing the low-speed compression damping circuit. In addition, a belt between the fork arch and the mechanical pivot of the cycle-ergometer was tightened as much as possible to avoid small vertical movement. The saddle height and the saddleto-handlebar distance were adjusted according to each participant's preference. Each cyclist used their own clipless pedals and cycling shoes.

The two pedalling exercises were performed at the same crank power output value (200 W for men and $175 \mathrm{~W}$ for women), and they were separated by 20 minutes of passive recovery. The cyclists were free to choose their pedalling cadence, but they could not vary it between the two exercises. They were instructed to maintain the target crank power output $( \pm 5 \mathrm{~W})$ and pedalling cadence $( \pm$ 2 rpm) continuously throughout the two pedalling exercises. This was important because any variation in pedalling cadence could induce changes in crank power output, as the braking resistance of the ergometer was always constant. Moreover, they were required to maintain the same hand position on the handlebar and degree of elbow flexion when the vibrations were applied. Each pedalling exercise included two 9-minute periods of pedalling with fork vibrations $(3 \times 3$ minutes) interspersed by 2 minutes of pedalling without fork vibrations. Six vibration frequencies were applied in the same order $(4,14,9,7,17,12 \mathrm{~Hz})$ every 3 minutes. Higher vibration frequencies than $20 \mathrm{~Hz}$ were not used because they were particularly unpleasant for the participants to grip the handlebar, even for short periods. 


\section{Measurements}

Power output $(\mathrm{W})$ and pedalling cadence (rpm) were measured every second by a wireless power meter (SRM Profesionnal model, Schoberer Rad Messtechnik, Fuchsend, Germany; crank length: $175 \mathrm{~mm}$; accuracy \pm $2 \%$ ) and stored by a Garmin watch worn by the participant (Forerunner 920 XT, Garmin Inc, Olathe, USA). Heart rate was measured every second with a cardio transmitter (Garmin premium heart rate monitor, Garmin Inc, Olathe, USA). The power meter was calibrated before each test according to the manufacturer's guidelines.

At the end of each three-minute pedalling bout with fork vibrations, the participants were instructed to report both their rating of perceived exertion (RPE) on the 6-20 Borg scale (Borg 1982) and their rating of perceived comfort (RPC) on a subjective scale from 0 (very high discomfort) to 10 (very high comfort) following a methodology similar to that reported by Millour et al. (2020). The value of 'five' on the latter scale corresponded to a neutral RPC.

Surface electromyography (EMG) activity of the flexor carpi radialis (FCR), extensor digitorum (ED), triceps brachii (TB), superior trapezius (ST), medial trapezius (MT) and erector spinae (ES) of the right upper limb was measured at $1926 \mathrm{~Hz}$ by wireless sensors (TrignoTM, Delsys Inc, Boston, USA), including two dry bar electrodes (1 ๑ $10 \mathrm{~mm}$ ) spaced $10 \mathrm{~mm}$ apart and three-axis accelerometers. According to the SENIAM (i.e., Surface Electromyography for the NonInvasive Assessment of Muscles project) recommendations, the EMG sensors were positioned on the middle of the muscle's belly and aligned in the direction of the muscle fibers (Hermens et al. 2000). The skin was shaved, rubbed with an abrasive paste (Nutriprep, AD Instrument, New South Wales, Australia), and cleaned with an alcohol swab. The EMG sensors were attached to the skin with a double-sided adhesive interface that matched the contour of the sensor and were secured by a medical adhesive (Transpore, 3M, Cergy, France). The EMG signals were recorded with a 16-bit amplifier system (TrignoTM Wireless Lab System, Delsys Inc.,
Boston, USA) with a gain of 1,000 and a common mode rejection of $80 \mathrm{~dB}$. All EMG signals were filtered with a band-pass filter $(10-500 \mathrm{~Hz})$ and were stored and analysed by a physiological data acquisition and analysis software (LabChart v8.1, AD Instruments, New South Wales, Australia). Muscular activity was quantified by the mean rootmean-square of the EMG signal computed over each 3-minute pedalling bout (RMSmean). All RMS values of each muscle were expressed in \% of the maximum RMS value measured during an isometric maximal voluntary contraction (MVC).

Wireless three-axis accelerometers (Hikob Fox, Hikob Agile France, Villeurbanne, France) with the synchronised data-logging mode were used to measure hand-arm transmitted vibration in accordance with the EN-ISO 53491 standard. The direction of the $\mathrm{x}$ axis was parallel to the horizontal plane of the bike, while the $y$ axis and the $z$ axis were perpendicular to the sagittal and horizontal planes of the bike, respectively (Figure 3 ). The accelerometers were placed on the mechanical pivot of the ergometer, on the top of the stem and on the top of the right wrist. The signals were sampled at $1,350 \mathrm{~Hz}$ with a full scale of \pm $24 \mathrm{~g}$. All data were logged, later transferred to a computer, converted to a hierarchal data format file (.h5) and processed using open source software for numerical computation (Scilab version 6.0, The MathWorks Inc, Natick, MA, United States). The EMG sensors were also used to measure vibrations on the muscles at $150 \mathrm{~Hz}$ with a full scale of $\pm 6 \mathrm{~g}$. One additional EMG sensor was placed on the ergometer pivot to determine the transmissibility of acceleration between the ergometer and each muscle. Acceleration signals from these sensors were recorded simultaneously with EMG signals and analysed with the same software. To quantify the amount of vibration the participants experienced, the effective amplitude of the total acceleration (aRMS) was calculated at the ergometer, the stem, the wrist and the muscles during each 3-minute pedalling bout from the square root of the sum of the RMS values squared for the acceleration measured along 
each axis (aRMS,i) in accordance with the ENISO 2631-1 standard:

$$
a_{R M S}=\sqrt{a_{R M S, X}^{2}+a_{R M S, Y}^{2}+a_{R M S, Z}^{2}}
$$

According to EN-ISO 5349, the RMS values of acceleration measured on the wrist and the muscles should be frequencyweighted because the risk of damage is not equal for all frequencies. Frequency weightings are designed so that they do not affect the frequencies at which the body is most sensitive and do attenuate the frequencies at which the response of the body is less sensitive. However, to simplify data processing, frequency weighting filter curves of the RMS values for the wrist and the muscles were not applied because it was assumed that the vibratory frequency affected the effective value of acceleration in the same manner during the two pedalling exercises. In addition, according to Mansfield (2005), the human responses to vibration are similar between 8 and $16 \mathrm{~Hz}$, which is close to the range of the frequency used in the current study (i.e., 4-17 Hz). Therefore, the idealized weighting curve was "flat", and the weighting factor was close to 1.00 . The reproducibility of vibrations delivered by the ergometer between the two experimental conditions was assessed by computing the coefficient of variation $(\mathrm{CV})$ of aRMs for each vibratory frequency and for each participant. The CV values were then averaged for all the vibratory frequencies and all the participants.

While assuming that the vibration movement was similar to a sine curve with one frequency, the peak-to-peak displacement $(\mathrm{D}$, $\mathrm{mm}$ ) of vibration measured on each axis was quantified on the basis of the peak acceleration (apeak) and the vibratory frequency:

(Eq. 2)

$$
a_{\text {peak }}=\frac{a_{R M S}}{\sqrt{2}}
$$

(Eq. 3)

$$
D=\frac{a_{\text {peak }}}{(2 \times \pi \times f)^{2} \times 1000}
$$

The vibration transmissibility ( $\mathrm{T}, \%)$ between the ergometer and the stem $\left(\mathrm{T}_{\text {stem//rgometer }}\right)$, that between the ergometer and the wrist ( $\left.T_{\text {wrist/ergometer }}\right)$ and that between the stem and the wrist $\left(\mathrm{T}_{\text {stem/wrist }}\right)$ were computed with the following equations:

(Eq.4)

$$
T_{\text {stem/ergometer }}=\frac{a_{R M S, \text { stem }}}{a_{R M S, \text { ergometer }}} \times 100
$$

$$
\begin{aligned}
T_{\text {writs/ergometer }}= & \frac{a_{R M S, \text { wrist }}}{a_{R M S, \text { ergometer }}} \times 100 \\
& \text { (Eq. } 6) \\
T_{\text {writs } / \text { stem }}= & \frac{a_{R M S, \text { wrist }}}{a_{R M S, \text { stem }}} \times 100
\end{aligned}
$$

The vibration transmissibility between the ergometer and each muscle ( $\mathrm{T}_{\text {muscle/rgometer }}$ ) was determined on the basis of the acceleration data measured with the EMG sensors and the following equation:

(Eq. 7)

$$
T_{\text {muscle/ergometer }}=\frac{a_{R M S, \text { muscle }}}{a_{R M S, \text { ergometer }}} \times 100
$$

The effectiveness of the fork suspension blocking was checked by computing the vibration transmissibility loss between the ergometer and the stem ( $\mathrm{T}_{\text {stem/ergome__loss, } \%)}$ by the following equation:

Tstem/ergometer_loss $=100 \%-$ Tstem/ergometer 
The handgrip force on the right side was measured with a grip force transducer (MLT 004/ST, AD Instruments, New South Wales, Australia) both one minute before and one minute after each pedalling exercise. The force signal was recorded with a 24-bit amplifier system (PowerLab 26T, AD Instruments, New South Wales, Australia) at a sampling frequency of $1,000 \mathrm{~Hz}$ and was stored by physiological data acquisition and analysis software (LabChart v7.4, AD Instruments, New South Wales, Australia). The participants were instructed to grip the force transducer as tightly as possible over a three-second period in a standing position while keeping their upper limb aligned with their trunk. Two trials interspersed by a minute of passive recovery were performed during each measurement period. The maximal grip force (MGF) corresponded to the maximum grip force value between the two trials. All the MGF values were expressed as \% of the MGF measured one minute before each pedalling exercise.

\section{Statistical analysis}

Statistical analysis was carried out using Past software (version 3.18, Øyvind Hammer, 276 Natural History Museum, University of Oslo, Norway). All data presented in the text, tables and figures correspond to the mean values \pm standard deviations (SDs) and $95 \%$ confidence intervals $(95 \% \mathrm{CI})$ of the mean differences. The mean values of EMG activity, vibration transmissibility, RPC and RPE were also computed for all vibratory frequencies for each experimental condition. The normality of the data distribution and homogeneity of the variances were assessed using the ShapiroWilk and Levene's tests, respectively. As some data did not met the normality and homogeneity criteria, non-parametric Wilcoxon signed rank tests were used to assess the differences in power output, pedalling cadence, heart rate, RPE, RPC, ergometer vibration (RMS acceleration and D values), vibration transmissibility and muscular activity between the two handle conditions and the differences in MGF between before and after each pedalling exercise. The level of significance was set to be $p<0.05$ for all tests. When significant differences were found, the effect size (ES) between two paired samples (Cohen, 1988) was calculated as the ratio of the mean to the SD in the differences of scores between EH and TH. As suggested by Cohen (1988), we defined effects as small (ES $<0.2)$, medium $(0.2<\mathrm{ES}<0.8)$ and large $(\mathrm{ES}>0.8)$. We considered that a large effect corresponded to substantial changes (Cohen, 1988).

\section{Results}

\section{Characteristics of ergometer vibration}

The average RMS value of the total acceleration measured at the pivot ergometer for all the vibratory frequencies was $22.8 \pm 12.5$ $\mathrm{m} / \mathrm{s}^{2}$ [range: $10.3-55.2 \mathrm{~m} / \mathrm{s}^{2}$ ] for TH and $24.2 \pm$ $14.5 \mathrm{~m} / \mathrm{s}^{2}$ [range: 10.3-55.8 m/s $\mathrm{s}^{2}$ ] for $\mathrm{EH}$. Independent of the handle condition, the RMS value of the total acceleration increased with the vibratory frequency. The reproducibility of the RMS value between the two pedalling exercises was higher for the $\mathrm{Z}$ axis $(\mathrm{CV}=0.2 \%)$ than for the $\mathrm{X}$ axis $(\mathrm{CV}=10.5 \%)$ and the $\mathrm{Y}$ axis $(\mathrm{CV}=5.9 \%)$. The $\mathrm{CV}$ of the RMS value of the total acceleration was $5.6 \%$. The average of the vibration peak-to-peak displacement (D) measured at the pivot ergometer for all the vibratory frequencies was $2.3 \pm 1.0 \mathrm{~mm}$ [range $1.7-4.2 \mathrm{~mm}$ ] for $\mathrm{TH}$ and $2.6 \pm 1.0 \mathrm{~mm}$ [range: 1.8-4.3 mm] for $\mathrm{EH}$ on the $\mathrm{X}$ axis, $1.6 \pm 1.0 \mathrm{~mm}$ [range: $0.5-2.7 \mathrm{~mm}$ ] for $\mathrm{TH}$ and $1.8 \pm 1.1 \mathrm{~mm}$ [range: $0.9-3.4 \mathrm{~mm}$ ] for $\mathrm{EH}$ on the $\mathrm{Y}$ axis, and $8.6 \pm 6.4 \mathrm{~mm}$ [range: $4.324 .6 \mathrm{~mm}$ ] for $\mathrm{TH}$ and $8.8 \pm 6.5 \mathrm{~mm}$ [range: $4.1-24.4 \mathrm{~mm}$ ] for $\mathrm{EH}$ on the $\mathrm{Z}$ axis. The peak-to-peak displacement measured on the $Z$ axis decreased as the vibratory frequency increased, while the $D$ values measured on the $X$ and $Y$ axes varied around their mean values with no clear trend.

\section{Cycling, physiological and subjective responses}

No significant differences in power output $(195 \pm 15 \mathrm{~W}$ vs. $195 \pm 16 \mathrm{~W})$, pedalling cadence ( $80 \pm 7 \mathrm{rpm}$ vs. $80 \pm 8 \mathrm{rpm})$, heart rate (111 \pm 12 bpm vs. $112 \pm 10 \mathrm{bpm}), \mathrm{RPE}(11.1 \pm 1.0$ vs. $11.3 \pm 1.1)$ and $\mathrm{RPC}(4.4 \pm 1.6$ vs. $4.0 \pm 1.5)$ 
were found between the two pedalling exercises performed with the $\mathrm{TH}$ and $\mathrm{EH}$, respectively.

\section{Handgrip force}

Figure 4 shows the MGF values measured before and after each pedalling exercise. Compared to the pre-exercise value, the postexercise MGF value significantly decreased with the $\mathrm{TH}$ by $7.2 \pm 7.2 \%(\mathrm{p}=0.007 ; \mathrm{ES}=1.01$; $95 \%$ CI: $4.0,11.3 \%$ ) but not with the EH.

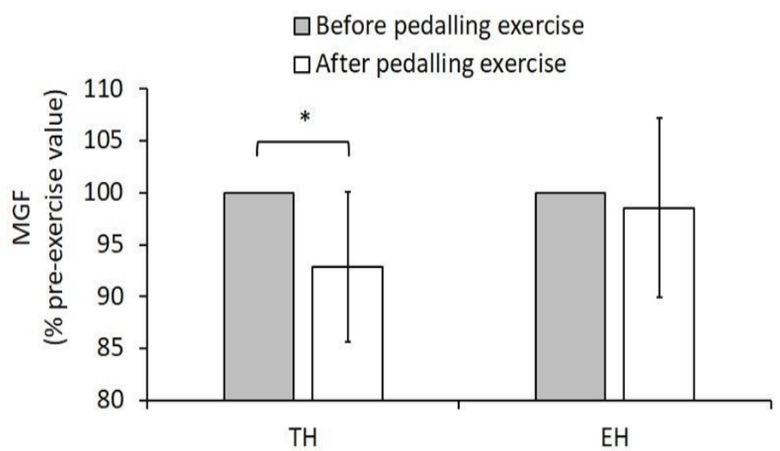

Figure 4. Maximal handgrip force (MGF) measured 1 minute after the pedalling exercise performed with the traditional handles (TH) and the ergonomic handles (EH) expressed as \% of MGF value measured 1 minute before the pedalling exercise. The star symbol indicates a significant difference between the two handle types: *: $p<0.05$

\section{Vibration transmissibility}

Figure 5 shows the vibration transmissibility of the total acceleration computed for all vibratory frequencies between the ergometer and the stem, between the stem and the wrist, and between the ergometer and the wrist, computed for all the vibratory frequencies and for each handle

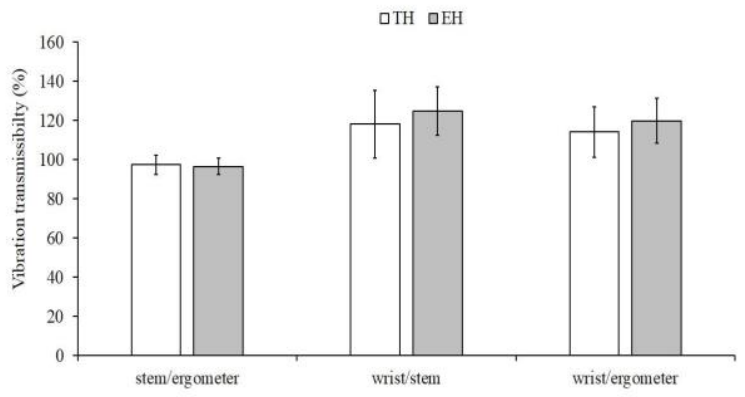

Figure 5. Mean values of vibration transmissibility between the ergometer and the stem, between the stem and the wrist, and between the ergometer and the wrist, measured with the traditional handles $(\mathrm{TH})$ and ergonomic handles $(\mathrm{EH})$ for all vibratory frequencies type. No significant differences in vibration transmissibility between the ergometer and the stem were found between the two handles, regardless of the vibratory frequency. Vibration loss between the ergometer and the stem was $3.0 \pm 4.7 \%$ for the two handles and for all participants and vibratory frequencies. Compared to the $\mathrm{TH}$, the $\mathrm{EH}$ involved for all vibratory frequencies a small increase in vibration transmissibility by $7.5 \pm 17.3 \%$ between the stem and the wrist and by $6.5 \pm$ $14.2 \%$ between the ergometer and the wrist, but these differences were not significant. However, the statistical analysis performed for each vibratory frequency showed that the EH induced a significant increase in vibration transmissibility between the stem and the wrist by $28.8 \pm 42.0 \%$ ( $p=0.046$; $E S=0.69 ; 95 \%$ CI: $6.0,51.6 \%$ ) and between the ergometer and the wrist by $25.6 \pm 32.3 \%(p=0.025 ; E S=0.79$; 95\% CI: 8.1, 43.2\%) at $17 \mathrm{~Hz}$. No significant difference between the two handles were found in the vibration transmissibility between the stem and the wrist and between the ergometer and the wrist for the 5 others vibration frequencies.

Figure 6 shows the vibration transmissibility of total acceleration between the ergometer and each upper body muscle, computed for all the vibratory frequencies and for each handle type. The transmissibility of total acceleration was always less than $70 \%$, regardless of the muscle and handle type. Compared to the $\mathrm{TH}$, the EH enhanced a lower vibration transmissibility in the ED (-10.4 \pm 9.1\%; $\mathrm{p}=0.002 ; \mathrm{ES}=-1.14,95 \% \mathrm{CI}:-14.8,-5.9 \%)$, FCR $(10.0 \pm 10.9 \% ; \mathrm{p}=0.003 ; \mathrm{ES}=-0.93,95 \% \mathrm{CI}$ : $-15.7,-4.7 \%)$ and TB $(-7.2 \pm 10.4 \% ; \mathrm{p}=0.02 ; \mathrm{ES}=$ $-0.69 ; 95 \%$ CI: $-12.3,-2.1 \%)$. The difference between the two handles was significant for the $\mathrm{ED}$ at 7, 9, 12 and $14 \mathrm{~Hz}$, with moderate to large effect sizes; for FCR at all frequencies except at $17 \mathrm{~Hz}$, with moderate to large effect sizes; and for $\mathrm{TB}$ at 7, 9 and $12 \mathrm{~Hz}$, with moderate to large effect sizes (Table 1). No significant differences between the two handles were found in the vibration transmissibility between the ergometer and the MT, ST or ES muscles (Figure 6). 


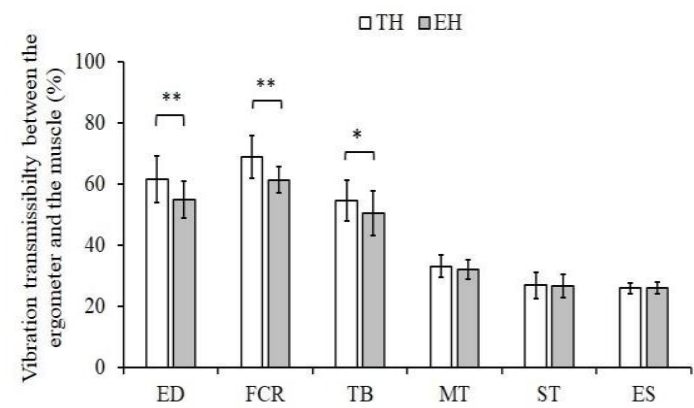

Figure 6: Mean values of vibration transmissibility between the ergometer and each upper body muscle measured with the traditional handles (TH) and ergonomic handles (EH) for all vibratory frequencies. ED: extensor digitorum; FCR: flexor carpi radialis; TB: triceps brachial; MT: median trapezius, ST: superior trapezius; ES: erector spinae. The star symbol indicates a significant difference between the two handle types: ${ }^{*}: p<0.05 ; * *: p<0.01 ;{ }^{* *}: p<0.001$.

\section{Muscular activity}

Figure 7 shows the average RMS value for all the vibratory frequencies for each upper body muscle and for each handle type. FCR activity decreased with the $\mathrm{EH}(-45.0 \pm 22.8 \%$; $\mathrm{p}=0.008 ; \mathrm{ES}=1.97 ; 95 \% \mathrm{CI}:-59.2,-30.9 \%)$, while $\mathrm{TB}$ activity increased with the $\mathrm{EH}(+11.5$ $\pm 22.4 \%, \mathrm{p}=0.033$; $\mathrm{ES}=0.51 ; 95 \%$ CI: -1.7 , $24.7 \%)$. The difference between the two handle types was significant at each vibratory frequency for FCR, with moderate to large effect sizes, but was significant for TB only at 9 and $17 \mathrm{~Hz}$, with moderate to large effect sizes (Table 2). No significant differences in ED, MT,

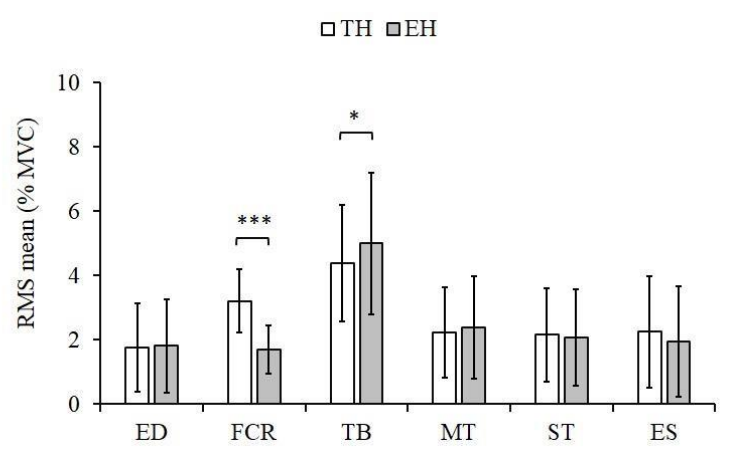

Figure 7. Muscular activity of each upper body muscle measured with the traditional handles (TH) and ergonomic handles (EH) for all vibratory frequencies. ED: extensor digitorum; FCR: flexor carpi radialis; TB: triceps brachii; MT: median trapezius, ST: superior trapezius; ES: erector spinae. All RMS mean values are normalized to the MVC values. The star symbol indicates a significant difference between the two handle types: *: $p<0.05 ;{ }^{* *}: p<0.001$.
ST or ES muscle activity were found between the two handles.

Table 1. Transmissibility of total acceleration (expressed in \%) between the ergometer and the extensor digitorum, flexor carpi radialis, triceps brachii and median trapezius muscles measured with the traditional handles (TH) and ergonomic handles $(E H)$ and ergonomic handles (EH) for each vibratory frequency. ES: effect size

\begin{tabular}{|c|c|c|c|c|}
\hline $\begin{array}{l}\text { Vibratory } \\
\text { frequenc } \\
\text { y }(\mathrm{Hz})\end{array}$ & $\mathrm{TH}$ & $\mathrm{EH}$ & $p$ value & ES \\
\hline \multicolumn{5}{|c|}{ Extensor Digitorum } \\
\hline 4 & $40.2 \pm 3.6$ & $40.0 \pm 3.6$ & 0.350 & 0.33 \\
\hline 7 & $56.8 \pm 6.9$ & $54.3 \pm 7.1$ & 0.006 & 0.76 \\
\hline 9 & $63.3 \pm 7.6$ & $57.1 \pm 6.5$ & $<0.001$ & 1.40 \\
\hline 12 & $77.1 \pm 17.9$ & $62.4 \pm 10.9$ & 0.002 & 1.20 \\
\hline 14 & $73.6 \pm 12.4$ & $62.6 \pm 11.9$ & 0.006 & 0.84 \\
\hline 17 & $58.3 \pm 11.6$ & $52.4 \pm 13.0$ & 0.097 & 0.31 \\
\hline \multicolumn{5}{|c|}{ Flexor Carpi Radialis } \\
\hline 4 & $43.5 \pm 4.0$ & $41.4 \pm 3.6$ & $<0.001$ & 1.62 \\
\hline 7 & $62.5 \pm 8.2$ & $58.4 \pm 5.7$ & 0.004 & 0.94 \\
\hline 9 & $73.1 \pm 10.5$ & $63.6 \pm 7.0$ & 0.002 & 1.32 \\
\hline 12 & $85.6 \pm 13.2$ & $67.9 \pm 8.6$ & 0.002 & 1.21 \\
\hline 14 & $79.8 \pm 11.4$ & $71.6 \pm 11.2$ & 0.055 & 0.54 \\
\hline 17 & $68.6 \pm 13.9$ & $64.9 \pm 13.5$ & 0.530 & 0.06 \\
\hline \multicolumn{5}{|c|}{ Triceps Brachii } \\
\hline 4 & $39.0 \pm 4.3$ & $39.2 \pm 3.5$ & 0.400 & 0.23 \\
\hline 7 & $60.9 \pm 12.1$ & $54.3 \pm 8.2$ & 0.004 & 0.96 \\
\hline 9 & $75.3 \pm 12.7$ & $62.6 \pm 12.8$ & $<0.001$ & 1.66 \\
\hline 12 & $71.4 \pm 15.9$ & $63.1 \pm 15.0$ & 0.009 & 0.77 \\
\hline 14 & $48.8 \pm 11.4$ & $49.0 \pm 12.5$ & 0.910 & 0.06 \\
\hline 17 & $31.8 \pm 6.4$ & $34.8 \pm 7.4$ & 0.087 & 0.45 \\
\hline
\end{tabular}




\section{Discussion}

Compared to $\mathrm{TH}$, (a) EH induced lower levels of vibration transmissibility in the forearm extensors and flexors and in the elbow extensor muscles for all the vibratory frequencies but increased vibration transmissibility to the wrist (only at $17 \mathrm{~Hz}$ ); (b) the muscular activity in the forearm flexors significantly decreased while the elbow extensor activity slightly increased; and (c) the $\mathrm{EH}$ did not involve a significant reduction in the maximal handgrip force after the pedalling exercise.

The participants were asked to perform the two pedalling exercises at the same absolute power output (175 W for women and $200 \mathrm{~W}$ for men) and at the same self-selected pedalling cadence. The participants were able to exert similar levels of effort, as evidenced by the nonsignificant differences between the two pedalling exercises in terms of power output, pedalling cadence, heart rate, and RPE. In view of these results and the same vibration stimuli between the two pedalling exercises (i.e., the RMS value of total acceleration reported above are very similar), differences in maximal grip force, vibration transmissibility and muscular activity were likely to be the result of the changes in the handle shape and in the handforearm position. Moreover, as we found no significant difference in vibration loss from the ergometer to the stem between the two experimental conditions, we cannot attribute the aforementioned differences to changes in damping at the front fork.

The first hypothesis of the current study was that EH induced both smaller vibrations and lower levels of muscle activity in the forearm and arm than did $\mathrm{TH}$, due to changes in the cyclists' hand position and forearm-arm configuration. It seems to be supported because muscle vibration dampening is higher with $\mathrm{EH}$ than with $\mathrm{TH}$. There were significant reductions in the vibration transmissibility of the total vibration to the wrist extensor (ED), wrist flexor (FCR), and elbow extensor (TB) muscles. The magnitude of decrease in vibration transmissibility was two to three times higher in the TB (-21\%) and in the FCR (-
$30 \%)$ than in the ED (-9\%). The lack of significant changes in the other muscles (i.e.,

Table 2. Table 2. Muscular activity of flexor carpi radialis and triceps brachii (RMS value expressed in \% MVC) measured with the traditional handles (TH) and ergonomic handles (EH) for each vibratory frequency. ES: effect size

\begin{tabular}{|c|c|c|c|c|}
\hline $\begin{array}{l}\text { Vibratory } \\
\text { frequency }(\mathrm{Hz})\end{array}$ & $\mathrm{TH}$ & $\mathrm{EH}$ & $\mathrm{p}$ value & ES \\
\hline \multicolumn{5}{|c|}{ Flexor Carpi Radialis } \\
\hline 4 & $2.3 \pm 2.1$ & $1.2 \pm 1.0$ & 0.047 & 0.94 \\
\hline 7 & $2.1 \pm 1.0$ & $1.0 \pm 0.5$ & 0.005 & 2.03 \\
\hline 9 & $2.6 \pm 1.2$ & $1.1 \pm 0.6$ & 0.007 & 2.28 \\
\hline 12 & $4.8 \pm 1.6$ & $2.9 \pm 1.3$ & 0.015 & 0.98 \\
\hline 14 & $3.9 \pm 1.5$ & $1.9 \pm 1.0$ & 0.017 & 0.95 \\
\hline 17 & $3.6 \pm 1.9$ & $2.1 \pm 0.9$ & 0.028 & 0.50 \\
\hline \multicolumn{5}{|c|}{ Triceps Brachii } \\
\hline 4 & $3.4 \pm 1.6$ & $3.2 \pm 1.6$ & 0.476 & 0.28 \\
\hline 7 & $3.9 \pm 1.8$ & $4.1 \pm 1.8$ & 0.185 & 0.22 \\
\hline 9 & $4.2 \pm 1.8$ & $6.5 \pm 3.1$ & 0.007 & 1.05 \\
\hline 12 & $4.6 \pm 2.2$ & $4.5 \pm 2.0$ & 0.721 & 0.07 \\
\hline 14 & $5.3 \pm 2.2$ & $6.1 \pm 2.7$ & 0.062 & 0.061 \\
\hline 17 & $5.0 \pm 2.2$ & $5.6 \pm 2.6$ & 0.018 & 0.55 \\
\hline
\end{tabular}

medial trapezius, superior trapezius and erector spinae) may be because the orientation of the trunk did not change when the EH were used instead of the TH. However, the first hypothesis is partly supported because we did not find any change in vibration transmissibility to the wrist. Moreover, as shown in Figure 7, the transmissibility of the total vibration between the ergometer and the wrist and between the stem and the wrist were slightly higher in the EH than in the $\mathrm{TH}$ at 17 $\mathrm{Hz}$. This effect could to be due to the change in the hand position on the handlebar with the $\mathrm{EH}$ because they are closer to the vertical axis of the fork than are the $\mathrm{TH}$. The increase in vibration transmissibility to the wrist with the $\mathrm{EH}$ might lead to an increased risk of damage on joint and ligaments. Consequently, future studies should evaluate the long-term effects of the use of EH on the health of cyclists.

Concerning muscular activity, compared to the $\mathrm{TH}$, the $\mathrm{EH}$ led to a larger reduction $(45 \%)$ in the EMG activity of the wrist flexor 
muscles (FCR) (Figure 9). This change was observed for all the vibratory frequencies, with substantial effect sizes. With the EH, the hands were simply resting on the medial carpus bone, and a large grip force was not required when the cyclists were pedalling with vibrations. Thus, the wrist flexor muscles were less active with the EH than with the TH. However, our first hypothesis was again partly confirmed, as the ergonomic handles slightly increased the EMG activity of TB $(+12 \%)$ for all vibratory frequencies, notably at 9 and $17 \mathrm{~Hz}$. The increase in elbow extensor activity may have been caused by the cyclists' arms being more outstretched with the $\mathrm{EH}$ than with the TH. Even though it was not measured, elbow extension could have slightly increased because of the changes in the hand position. The EMG activity of the other muscles (i.e., ED, MT, ST and ES) did not significantly change with the EH compared with the TH. To our knowledge, only two studies (Arpinar-Asvar et al. 2013; Hurst et al. 2012) have reported the EMG activity of upper body muscles while participants pedalled with vibrations. ArpinarAsvar et al. (2013) measured the EMG activity of the FCR and ED muscles while participants cycled on different road surfaces (i.e., smooth road, concrete stone pavement and rough road) using road and mountain bikes. The authors found larger EMG values for the FCR $(12.5-27.1 \%$ of MVC) and ED (14.7-49.1\% of MVC) muscles during the MTB experimental conditions than we did in the current study (< $5 \%$ of MVC). These differences may have been due to differences in (i) the method used for obtaining the reference value for the EMG normalization (i.e., MVC gripping tasks vs. classical MVC), (ii) the effort required for the task by the participants (i.e., cycling outdoors without pedalling but controlling the handlebars vs pedalling indoors without bike handling), and (iii) the range of the effective value of the vibrations (i.e., $1.5-9.7 \mathrm{~m} / \mathrm{s}^{2}$ vs. 10.4-50.0 m/ $/ \mathrm{s}^{2}$ ). Hurst et al. (2012) compared the muscle activity of the upper body in elite cross-country and downhill mountain bikers during off-road downhill cycling. As did Arpinar-Avsar et al. (2013), the authors reported higher normalized EMG activity in the TB, which always exceeded $20 \%$ of CMV, than we did in the current study ( 5\%). More work was done by the upper body muscles in their study than in the current study with regard to piloting the bike, supporting the body weight, and absorbing large vibrations and shocks in the field.

Our second hypothesis is supported since the handgrip force measured immediately after the pedalling exercise with the EH was significantly unchanged while it was decreased approximately by $5 \%$ after the pedalling exercise with the EH. Although the underlying physiological mechanisms remain unclear, the decrease in handgrip force may be related to ulnar tunnel syndrome (Akuthota et al. 2005), which is caused by misalignment of the hand and forearm while using cylindrical handles. Unlike the $\mathrm{TH}$, the $\mathrm{EH}$ allowed the maximal handgrip force values to be maintained after the pedalling exercise. This result could indicate that $\mathrm{EH}$ compress nerves less than TH do. It is likely that this positive and beneficial effect is related to the decrease in EMG activity in the FCR muscle. The motor units of the wrist flexor muscles were probably less active at the end of the pedalling exercise performed with the EH than at the end of the exercise performed with the TH. Therefore, the central nervous system could increase the firing rate and recruit additional motor units to produce a similar maximal handgrip force after the pedalling exercise compared with before the exercise.

The present study has four limits that must to be considered. Firstly, the nature of the vibrations produced by the ergometer (i.e., sine curves with one vibratory frequency) did not exactly mimic the actual vibrations incurred during mountain biking because both the amplitude and the frequency vary continuously in the field and depend on the roughness of the terrain and the obstacles (Hurst et al. 2012; Macdermid et al. 2014). Therefore, the results may have differed if this study was conducted under field conditions. However, the vibration characteristics during the current study were controlled, which could not have been possible if the study was conducted under field conditions. In addition, the range of vibratory frequencies studied (4- 
$17 \mathrm{~Hz}$ ) is very close to the range of maximum vibratory frequency $(7-12 \mathrm{~Hz})$ measured at the handlebar during uphill and downhill sections of an XC track performed at race pace-level effort (Macdermid et al. 2014). The second limit is that the human response to vibration with the EH may have been different if the measurements were performed with a suspension fork inflated with opening of lowspeed compression damping circuit (as recommended by the fork manufacturer guidelines). In this case, vibration damping would be much more important, and thus, the effect of EH could perhaps be reduced. The third limit is that vibration transmissibility between the ergometer and muscles could be underestimated because the accelerometers were placed on soft tissue which could damp vibrations, instead of hard tissue (i.e. bones) as it is generally preconised (EN-ISO 5349-1 standard). However, since the sensor locations were not changed between the two handles condition, we can suppose that this factor had same influence on the accelerometry data while the two pedalling exercises. The four limitation is that we assessed only the acute effect of $\mathrm{EH}$ on the human response to vibrations, and we compared it only to that of TH. Thus, it might be possible that changes in some variables would be different if the participants use the EH for several weeks or months. It would be also interesting to compare the effect of $\mathrm{EH}$ to the ergonomic cylindrical handles that offer a large support on the lateral side.

\section{Practical Applications.}

The present findings showed that SPIRGRIPS ${ }^{\circledR}$ handles decreased both the vibration transmissibility and the muscular activity in the forearm muscles during a pedalling exercise with vibrations when compared to traditional cylindrical handles. Moreover, unlike the traditional cylindrical handles, the ergonomic clip on handles did not lead to a significant reduction in the maximal handgrip force after the pedalling exercise. Considering these results, the use of SPIRGRIPS@ could potentially reduce the symptoms of ulnar tunnel syndrome and hand-arm vibration syndrome in mountain bikers, but this hypothesis should be tested by future clinical studies. In addition, it would be also interesting to study in the future the influence of other ergonomic clip on handles (e.g. SQlab Innerbarends ${ }^{\circledR}$ ) or shock absorber handlebars (e.g. BARAMIND ® MTB handlebar) on the vibration transmissibility and muscular activity in order, on the one hand, to corroborate the results found in this present study and, on the other hand, to compare their effectiveness on damping vibrations to that of SPIRGRIPS ${ }^{\circledR}$.

Even though we did not investigate the potential influence of the ergonomic clip-on handles on MTB performance, using them instead of the TH during the easier sections of a track (nontechnical trails) could enable mountain bikers to reduce the fatigue of forearm muscle, and thus, preserve their handgrip strength for effective braking during steep downhill trails. While it is possible for cyclists to brake when their hands are on the $\mathrm{EH}$, their braking ability could be influenced by the $\mathrm{EH}$ because the fingers operate on the brake levers in a different way. Future studies should evaluate the effect of EH on MTB performance and their influence on braking ability.

\section{Acknowledgments:}

The authors thank Pascal Badollet for his technical assistance and Frederyck Dutent for his help in writing the article in English.

\section{Conflicts of Interest:}

The authors declare that they received no funding from the ergonomic clip-on handle company and that they have no conflicts of interest regarding the results of the current study.

\section{References}

1. Abercromby AFJ, Amonette WE, Layne CS, McFarlin BK, Hinman MR, Paloski WH (2007) Variation in neuromuscular responses during acute whole-body vibration exercise. Medicine \& Science in Sports \& Exercise 39(9):1642-1650. DOI: $10.1249 / \mathrm{mss} .0 \mathrm{~b} 013 \mathrm{e} 318093 \mathrm{f} 551$

2. Akuthota V, Plastaras C, Lindberg K, Tobey J, Press J, Garvan C (2005) The effect of longdistance bicycling on ulnar and median nerves: an electrophysiologic evaluation of 
cyclists palsy. The American Journal of Sports Medicine 33(8):1224-1230. DOI: 10.1177/0363546505275131

3. Aldien $\mathrm{Y}$, Marcotte P, Rakheja S, Boileau PE (2005) Mechanical impedance and absorbed power of hand-arm under $\mathrm{x}(\mathrm{h})$-axis vibration and role of hand forces and posture. Industrial Health 43(3):495-508. DOI: 10.2486/indhealth.43.495

4. Arpinar-Asvar P, Brilik G, Sezgin OC, Soylu AR (2013) The effects of surface-induced loads on forearm muscle activity during steering a bicycle. Journal of Sports Science and Medicine 12:512-520.

5. Bertucci, W, Chiementin X (2013) Solutions to reduce vibratory stress generated on the handarm system in cycling: preliminary study. In: Proceedings of the $15^{\text {th }}$ international congress of French researchers association in Sport and Physical Activity.

6. Borg GA (1982). Psychophysical bases of perceived exertion. Medicine \& Science in Sports \& Exercise 14:377-381.

7. Capitani D, Beer S (2002) Handlebar palsy- a compression syndrome of the deep terminal (motor) branch of the ulnar nerve in biking. Journal of Neurophysiology 249:1441-1445. DOI: $\underline{10.1007 / s 00415-002-0864-4}$

8. Cohen J (1988) Statistical power analysis for the behavioural sciences. Routledge

9. De Lorenzo DS, Hull ML (1999) Quantification of structural loading during off-road cycling. Journal of Biomechanical Engineering 121(4):399-405. DOI: $\underline{10.1115 / 1.2798337}$

10. De Ponte ÁM, Guirro EC, Pletsch AH, DibaiFilho AV, Brandino HE, Guirro RR (2015) The forearm positioning changes electromyographic activity of upper limb muscles and handgrip strength in the task of pushing a load cart. Journal of Bodywork and Movement Therapies 19(4):597-603. DOI: 10.1016/j.jbmt.2014.09.006

11. Di Domizio J, Keir PJ (2010) Forearm posture and grip effects during push and pull tasks. Ergonomics 53(3):336-43. DOI: $\underline{10.1080 / 00140130903389076}$

12. Faiss R, Praz M, Meichtry A, Gobelet C, Deriaz $O$ (2007) The effect of mountain bike suspensions on vibrations and off-road uphill performance. Journal of Sports Medicine \& Physical Fitness 47:151-158.

13. Harris DJ, Atkinson G (2011) Update-Ethical standards in sport and exercise science research. International Journal of Sports Medicine 32(11):819-821. DOI: 10.1055/s-0031$\underline{1287829}$

14. Hazell TJ, Jakobi JM, Kenno KA (2007) The effects of whole-body vibration on upper- and lower body EMG during static and dynamic contractions. Applied Physiology Nutrition and Metabolism 32(6):115-1163. DOI: $\underline{10.1139 / \mathrm{H} 07-116}$

15. Hermens HJ, Freriks B, Disselhorst-Klug C, Rau G (2000) Development of recommendations for SEMG sensors and sensor placement procedures. Journal of Electromyography and Kinesiology 10(5):1156-1163. DOI: $\quad$ 10.1016/s10506411(00)00027-4

16. Hurst $H$, Swarén $M$, Hébert-Loisier $K$, Ericsson F, Sinclair J, Atkins S, Holmberg HC (2012) Influence of course type on upper body muscle activity in elite cross-country and downhill mountain bikers during off road downhill cycling. Journal of Science \& Cycling 1(2):2-9.

17. International Organization for Standardization (1997) Mechanical vibration and shock-Evaluation of human exposure to whole-body vibration - Part 1: General requirements. EN-ISO 2631-1. Geneva, Switzerland.

18. International Organization for Standardization (2001) Mechanical vibration Measurement and evaluation of human exposure to hand-transmitted vibration -Part 1: General Requirements. EN-ISO 5349-1. Geneva, Switzerland.

19. Issurin VB (2005) Vibrations and their application in sport. A review. J Sports Med Phys Fitness 45:324-336.

20. Lee H, Martin DT, Anson JM, Grundy D, Hahn AG (2002) Physiological characteristics of successful mountain bikers and professional road cyclists. Journal of Sports Sciences 20(12):1001-1008.

DOI: $\underline{10.1080 / 026404102321011760}$

21. Levy M, Smith, GA (2005) Effectiveness of vibration damping with bicycle suspension systems. Sports Engineering 8:99-106.

22. Macdermid PW, Fink PW, Stannard SR (2014) Transference of 3D accelerations during cross country mountain biking. Journal of Biomechanics 47(8):1829-1837. DOI: 10.1016/j.jbiomech.2014.03.024

23. Macdermid PW, Miller MC, Macdermid FM, Fink PW (2015) Tyre volume and pressure 
effects on impact attenuation during mountain bike riding. Shock and Vibration 1-10.

24. Mansfield NJ (2005) Human response for vibration. CRC Press LCC, Boca Raton

25. Mester J, Spitzenfeil P, Schwarzer J, Seifriz J (1999) Biological reaction to vibrationimplication for sport. Journal of Science and Medecine in Sport 2(3):211-226. DOI: $\underline{10.1016 / \mathrm{s} 1440-2440(99) 80174-1}$

26. Millour G, Duc, S, Puel F, Bertucci W (2020) Effect of asymmetric crank arm lengths on performance-related variables in cyclists with an anatomical lower limb length discrepancy. Sports Engineering, 23(1): 1-9. DOI: 10.1007/s12283-020-00327-w

27. Radwin RG, Arsmtrong TJ, Chaffin DB (1987) Power hand tool vibration effects on grip exertions. Ergonomics 30:833-855.

28. Steward JD (1993) Ulnae neuropathies at the wrist and the hand. In: Steward JD (ed). Focal peripheral neuropathies. Raven Press, New York, 220-225.

29. Tarabini, M, Saggin B, Scaccabarozzi D (2015) Whole-body vibration exposure in sport: four relevant cases. Ergonomics 58(7):11431150. DOI: $10.1080 / 00140139.2014 .961969$

30. Wakeling JM, Nigg BM, Rozitis AI (2002) Muscle activity damps the soft tissue resonance that occurs in response to pulsed and continuous vibrations. Journal of Applied Physiology 93(3):1093-1103. DOI: 10.1152/japplphysiol.00142.2002

31. Wang EL, Hull ML (1997) A dynamic system model of an off-road cyclist. Journal of Biomechal Engineering 119(3):248-253. DOI: $\underline{10.1115 / 1.2796088}$

32. Xu SX, Dong GR, Welcome DE, Warren C, McDowell TW, Wu JZ (2017) Vibrations transmitted from human hands to upper arm, shoulder, back, neck, and head. International Journal of Industrial Ergonomics 62:1-12. DOI: $\underline{10.1016 / j \text {.ergon.2016.07.001 }}$ 